\title{
On the role of confinement on solidification in pure materials and binary alloys
}

\author{
B. P. ATHREYA ${ }^{\dagger}$, J. A. DANTZIG ${ }^{\dagger}$, S. LIU ${ }^{\ddagger}$, and R. TRIVEDI ${ }^{\ddagger}$ \\ ${ }^{\dagger}$ Department of Mechanical and Industrial Engineering, \\ University of Illinois, Urbana, IL 61801. \\ ${ }^{\ddagger}$ Department of Materials Science and Engineering, \\ Iowa State University, Ames, IA 50011.
}

\begin{abstract}
We use a phase-field model to study the effect of confinement on dendritic growth, in a pure material solidifying in an undercooled melt, and in the directional solidification of a dilute binary alloy. Specifically, we observe the effect of varying the vertical domain extent $(\delta)$ on tip selection, by quantifying the dendrite tip velocity and curvature as a function of $\delta$, and other process parameters. As $\delta$ decreases, we find that the operating state of the dendrite tips becomes significantly affected by the presence of finite boundaries. For particular boundary conditions, we observe a switching of the growth state from $3-\mathrm{D}$ to $2-\mathrm{D}$ at very small $\delta$, in both the pure material and alloy. We demonstrate that results from the alloy model compare favorably with those from an experimental study investigating this effect.
\end{abstract}

Keywords: Dendrites, Phase field, Adaptive grid, Confined growth

\section{INTRODUCTION}

Dendrites are one of the basic microstructural patterns seen in solidified metals. The mechanical behavior of the solidified product is often decided by the length scales set by these patterns. Study of dendritic growth is therefore motivated by the need to predict these length scales. The fundamental quantities that completely describe the growth of a dendrite at steady state under a given set of external conditions are its tip velocity and radius, which together define the so called "operating state". Despite considerable advances in the understanding of solidification science, discrepancies still arise when one attempts to compare theoretical predictions of dendrite operating states with experimental observations. We find that some of these discrepancies derive from differences between the ideal conditions assumed in theoretical treatments, and those experienced by materials under actual experimental situations.

The first theoretical treatment of the "free" dendrite growth problem was presented by Ivantsov [1]. He considered a pure dendrite, modeled as a paraboloid of revolution with tip curvature $\rho_{\text {tip }}$, growing into an infinite undercooled melt with temperature $T \rightarrow T_{\infty}$ far from the advancing tip. The dendrite was assumed to be isothermal at the melting temperature $T_{m}$, and to be growing along its axis at constant velocity $V_{t i p}$ in a shape-preserving way. Ivantsov found a solution to the thermal transport problem, in which the dimensionless undercooling $\Delta=\left(T_{m}-T_{\infty}\right) /\left(L_{f} / c_{p}\right)=\mathcal{I}(P e)$ where $P e=\rho_{t i p} V_{t i p} / 2 D$ is the Péclet number, $D$ is the thermal diffusivity, and $\mathcal{I}$ is the Ivantsov function. The temperature has been scaled by the characteristic temperature $L_{f} / c_{p}$, with $L_{f}$ being the latent heat of fusion and $c_{p}$ the specific heat.

This solution presented a conundrum, because it showed that the transport problem alone did not uniquely specify the operating state of the dendrite, i.e., the single combination of $\rho_{t i p}$ and $V_{t i p}$ observed in experiments. Additional considerations, such as the effect of curvature on the melting point [2], stability [3, 4] and eventually the anisotropy of the surface tension [5, [6, 7], led to a second condition $\sigma^{*}=2 d_{0} D / \rho_{\text {tip }}^{2} V_{t i p}$ where $\sigma^{*}$ is called the selection constant, and $d_{0}$ is the capillary length. The combination of Ivantsov's solution (modified for surface tension and its anisotropy) and the condition $\sigma^{*}$ is constant gives a unique operating state. Numerical simulations using the phase-field method [8] at large values of $\Delta$, have found agreement with the predictions of this body of work, known as microscopic solvability theory.

Glicksman and co-workers developed experimental techniques for studying the solidification of pure materials, with the objective of observing the operating state. They performed experiments with phosphorous [9], and transparent analog alloys like succinonitrile (SCN) [10] and pivalic acid (PVA) [1]. The results of these careful experiments found some areas of agreement with microscopic solvability theory, in particular, the value of $\sigma^{*}$ was found to be constant, but the operating combination of $\rho_{t i p}$ and $V_{t i p}$ did not agree. Provatas, et al. were able to explain this discrepancy by showing that for the low undercooling conditions found in the experiments, interaction between neighboring dendrite branches [12, 13] affected the operating state.

Experiments have also been performed to examine the role of superimposed fluid flow on dendritic growth. Gill and coworkers [14, 15] used SCN in a special cylindrical chamber with a bellows to effect fluid flow. Bouissou and Pelcé [16] performed experiments with a flowing alloy of PVA and a seed confined between microscope slides. Saville and Beaghton [17] presented a theoretical analysis which extended Ivantsov's solution to consider the superimposed flow. Jeong et al. [18] performed phase-field simulations of these experiments, and once again found discrepancies 
with theory. They conjectured that the differences arose because of the effect of finite containers in the experiments, leading to boundary conditions which differed from the assumptions of infinite media used in the theory.

Dendrite tip theories for constrained growth, such as directional solidification of dilute binary alloys between microscope slides, have been developed by Trivedi [19] and, Kurz and Fisher [20]. They have shown that a relationship of the form $\rho_{t i p}^{2} V_{t i p}=$ constant, should hold for constrained growth just as in free dendrite growth. Early experiments by Somboonsuk et al. 21] in samples with slide separation greater than $150 \mu \mathrm{m}$ have shown excellent agreement with this theory. However, in recent studies Liu et al. 22] have demonstrated that experimental results start to deviate significantly from theory when the slide separation approaches the scale of the primary dendrite spacing.

In this article, we systematically study the role of confinement on dendritic growth. Since every experiment is performed in a finite container, we feel that this effect cannot be ignored. The first two authors have previously reported a study on confined growth in pure materials [23]. Here we extend our investigations to directionally solidified binary alloys. For purposes of continuity and completeness, we will again present results from our study on pure materials. For the binary alloy, recent experimental data from Liu et al.[22], will provide us with an avenue for testing our numerical predictions.

\section{MODELING}

\section{A. Phase field model, adaptive grids and numerical methods}

The objective in a general solidification problem is to solve the equations governing thermal and solute transport, subject to boundary conditions on the solid-liquid interface (moving boundary) and elsewhere. If melt convection is to be modeled, one needs to solve the momentum equations for fluid flow simultaneously with the above transport equations. Imposing the interface boundary conditions upon discretizing the governing equations poses a difficulty however, since the interface, as it evolves, will not in general align itself with a fixed set of mesh points.

The phase-field method eliminates the sharp liquid-solid boundary by introducing evolution equations for a continuous order parameter $\phi \in[-1,1]$, where $\phi=-1,+1,0$ corresponds to liquid, solid and interface respectively. Thus, the arduous task of solving the transport equations separately in liquid and solid domains while simultaneously satisfying boundary conditions on arbitrarily shaped interfaces, is replaced by that of solving a system of coupled differential equations; one for the evolution of $\phi$ and one for each of the transport variables (temperature, concentration and velocity). Phase-field modeling has been an active area of research in the past decade, and we refer the interested reader to original work by Langer [24], Karma and Rappel [8], and Beckermann et al. 25] for derivations of the phase-field equations and selection of phase-field parameters ensuring convergence to the original sharp interface problem.

The phase-field model introduces a parameter $W_{0}$ that connotes the finite width of the now 'smeared' interface. Karma and Rappel [8] showed that the model converges to the sharp interface equations when $p=W_{0} V_{t i p} / D \ll 1$, where $D$ is the thermal or concentration diffusion coefficient, and $V_{t i p}$ is the nominal tip velocity of the dendrite. Resolving the interface on a discrete mesh requires that the mesh spacing $\Delta x \sim W_{0}$, while demanding that the diffusion field not interact with the boundaries leads to the domain size $L_{B} \gg D / V_{t i p}$. Satisfying these requirements causes calculations on regular meshes to quickly reach the limit of available computing resources. For example, if we choose $p=0.01$, fix $L_{B} V_{\text {tip }} / D=10$, and enforce $\Delta x=W_{0}$, then we find that the number of grid points per dimension on a regular mesh should be at least $L_{B} / W_{0}=1000$. This makes computations challenging on regular meshes even in 2-D, while 3-D computations may not be practical at all, depending on available computing power.

We have mitigated this problem successfully by solving the equations on an adaptive finite element mesh [13, 18]. In three dimensions, we use eight-noded trilinear brick elements stored using an octree data structure. A local error estimator indicates refinement or coarsening of the mesh, and this permits tracking of the interface as well as resolution of gradients in the other fields. There are six degrees of freedom at each node (three velocities, pressure, temperature/concentration and $\phi$ ), and a typical computation reaches well over one million unknowns. The finest elements $\left(\Delta x_{\min }\right)$, which are distributed near the interface, now need to be order of $W_{0}$.

For our studies on pure materials, we have used a finite element discretization of the 3-D phase field model developed by Karma and Rappel [8]. In order to account for the effects of melt convection we adopt the formulation presented by Beckermann et al. [25], who use an averaging method for the flow equations coupled to the phase-field. By appropriate choice of phase-field parameters we have ensured zero interface kinetics, which is a valid assumption for the range of undercooling we are concerned with.

For our alloy simulations, we have used a one sided (vanishing solid diffusivity) phase-field model [26, 27, 28], with a frozen temperature approximation. In a directional solidification arrangement, for certain values of the problem parameters (particularly when simulating real materials), a considerable amount of time can elapse before the transients vanish and the solid-liquid interface reaches steady state. In that time, the interface can encounter the end of the simulation box if the equations are solved in a reference frame that is fixed globally, and if the box is not large enough 
to contain the diffusion field. To alleviate this difficulty, we have solved the phase-field equations in a coordinate frame translating with the pulling speed. This saves some computational expense by allowing us the use of smaller boxes. We have not investigated the effect of melt convection in our numerical experiments with alloys.

In a recent article, Echebarria et al. [28] have emphasized that the same choice of phase-field parameters that produced zero interface kinetics in the pure material cannot also ensure this condition in the alloy model. This is due to the presence of certain additional terms in the kinetic parameter $\beta$, that arise out of accounting for the discontinuity in the concentration field at the interface in a model with vanishing solid diffusivity. To ensure that the kinetic coefficient is negligible at the interface, the phase-field relaxation time $\tau$ needs to be made temperature dependent in this region by setting

$$
\tau=\tau_{0}\left[1-(1-k) \frac{z-V_{p} t}{l_{T}}\right]
$$

Here $\tau_{0}$ is the usual relaxation time, $k$ is the partition coefficient, $z$ is the distance from the interface, $V_{p}$ is the pulling velocity, $t$ is time and $l_{T}$ is the thermal length [26].

We evolve the nonlinear order parameter equation using a Forward-Euler time stepping scheme, while the linear thermal/solute transport equations are solved using the Crank-Nicholson scheme with a diagonally preconditioned conjugate gradient solver. The transport equations typically converge in fewer than five iterations per time step. The 3-D flow equations for the pure material are solved using the semi-implicit approximate projection method (SIAPM) 29]. Details of the above numerical methods and the finite element formulation are omitted here, as they have been presented elsewhere [18].

\section{B. Geometry, initial and boundary conditions}

Our three dimensional simulation domain is the rectangular parallelepiped illustrated in Fig. 1 with edge lengths along the $x, y$ and $z$ axes; $L_{x}, L_{y}$ and $\delta$ respectively. The edges of the box are oriented along $\langle 100\rangle$ cubic crystallographic directions.

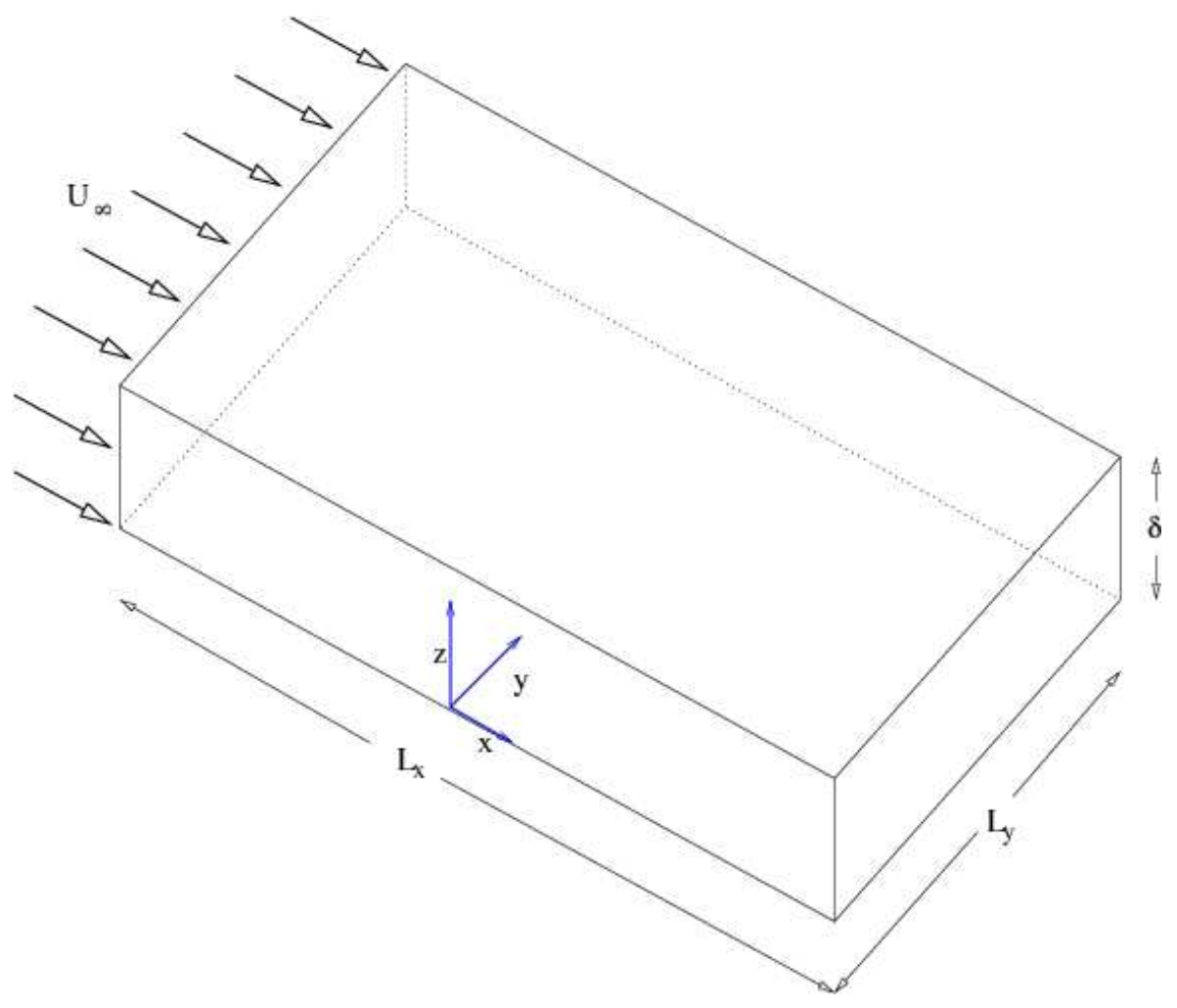

FIG. 1: Simulation Domain. All surfaces are modeled as symmetry planes in pure material simulations, whereas the surfaces $y=0$ and $y=L_{y}$ are periodic boundaries in the alloy simulations. 
Table 1. Pure material simulations

\begin{tabular}{llll}
\hline \hline Case & $\Delta$ & $U_{\infty}$ & $\delta$ \\
\hline $1(\mathrm{a})-(\mathrm{f})$ & 0.55 & 0 & $128,64,32,16,8,4$ \\
$2(\mathrm{a})-(\mathrm{f})$ & 0.55 & 5 & $128,64,32,16,8,4$ \\
$3(\mathrm{a})-(\mathrm{e})$ & 0.25 & 0 & $128,64,32,16,8$ \\
$4(\mathrm{a})-(\mathrm{f})$ & 0.25 & 5 & $128,64,32,16,8,4$ \\
$5(\mathrm{a})-(\mathrm{d})$ & 0.15 & 0 & $128,64,32,16$ \\
$6(\mathrm{a})-(\mathrm{e})$ & 0.15 & 5 & $128,64,32,16,8$ \\
\hline
\end{tabular}

For the pure material, the initial condition is a spherical solid seed with a radius greater than the critical nucleation radius, centered at the origin depicted in Fig. 11 Because of the inherent symmetry in the growth of the seed, it is usually sufficient to model one octant in three dimensional space. However, if forced fluid convection is incorporated along a particular direction, then the solidification rates into and counter to this direction become unequal. For example, if there is a flow parallel to the $x$ axis, $x=0$ is no longer a plane of symmetry. To account for this break in symmetry, we have to model at least a quadrant of space, with $z=0$ and $y=0$ as planes of symmetry.

In the simulations with the pure material, the dimensionless thermal field $u$ is subjected to zero flux $(\nabla u \cdot \mathbf{n}=0)$ boundary conditions on all surfaces. Fluid flow, when it is included in our study, is imposed as an inlet boundary condition $U_{\infty}$, normal to the face $x=-L_{x} / 2$. The velocity field is subjected to symmetry boundary conditions on the domain walls, and is forced to vanish in the solid $(\phi=1)$ by an appropriate formulation of the momentum equations (see 25]). We fix the lateral dimensions of the simulation box ( $L_{x}=512$ and $L_{y}=256$ are typical values), and study the interface evolution as a function of $\delta$, which is varied from 128 to 4 . Here, $L_{x}, L_{y}$ and $\delta$ are in units of the interface width $W_{0}$. For very small $\delta(\leq 8)$, steady growth conditions are reached relatively quickly for large undercooling. To save on computational cost in these runs (where $\Delta x_{\min }=0.5$ ), we sometimes use shorter lengths for $L_{x}$ and $L_{y}$, chosen to ensure that the diffusion field does not interact with the ends (in the $x$ and $y$ directions) of the box. For smaller $\Delta$ however, it typically takes much longer to reach steady conditions, and when melt convection is included, it can take impractically long CPU times to get converged results. For these cases, we terminate our runs when the tip radius/velocity versus time curves start to even out. Fortunately, it turns out that the behavior we are interested in appears for combinations of $\delta$ and $\Delta$ where steady state conditions are always achieved.

In the alloy simulations, the initial condition is a planar interface at $x=X_{0}$, perturbed by randomly spaced finite amplitude fluctuations. The box in these simulations is taken to represent the shallow channel between microscope slides where directional solidification conditions are imposed, viz. a fixed thermal gradient moving at a constant speed $V_{p}$. Once again, in this arrangement we study the influence of the depth of the channel $\delta$ (or equivalently the sample film thickness), on interface morphology. To minimize the diffusion field's interaction with the lateral boundaries, $L_{x}$ and $L_{y}$ are chosen to be relatively large $(\sim 256)$. We enforce zero flux boundary conditions on the concentration field, on the surfaces $x=L_{x} / 2=-L_{x} / 2$ and $z=0=\delta$, while periodic boundary conditions are imposed on the boundaries $y=0$ and $y=L_{y}$. The rationale behind periodic boundary conditions is to be able to simulate an infinite domain in $y$.

Unless otherwise stated, on each boundary, we employ the same type of boundary condition on the phase-field variable $\phi$, as we do on the transport variable. Where $\nabla \phi \cdot \mathbf{n}=0$, the material "wets" the boundaries, and the corresponding contact angle is $90^{\circ}$. Sémoroz et al. have previously used this technique to capture wetting of solid surfaces, with a two-dimensional phase-field model for binary alloys [30]. We also show a calculation with $\phi=-1$ on the boundaries, which is equivalent to making the material "non-wetting" (contact angle $=0^{\circ}$ ). The real contact condition probably lies somewhere in between these two extremes.

\section{EFFECT OF SMALL $\delta$ IN A PURE MATERIAL}

In this section, we report the effect of changing $\delta$ on the tip of a pure material dendrite, evolving along the negative $x$ axis (upstream direction when flow is present). We simulated the cases shown in Table 1 . We used a fixed value for the four-fold anisotropy in all our simulations $\left(\epsilon_{4}=0.05\right)$. We have not corrected for grid anisotropy $[8]$ in these calculations, but work at a grid spacing where its effect is known to be small [13].

We use the following values for parameters in our calculations: interface width $W_{0}=1$, time scale for interface kinetics $\tau_{0}=1$, coupling constant $\lambda=6.383$, thermal diffusivity $D=4$, capillary length $d_{0}=0.1385 W_{0}$ (which leads to zero interface kinetics), and Prandtl number $\operatorname{Pr}=23.1$, where $W_{0}, \tau_{0}$ and $D$ are in dimensionless units (see [8]). 


\section{A. "Wetting" boundary conditions, $\nabla \phi \cdot \mathbf{n}=0$}

To make ideas more concrete, we choose cases 3 and 4 as a representative subset of our computations and present detailed analyses on those runs. Figures 2 and 3 show the upstream dendrite's tip velocity and radius respectively, as functions of the box height $\delta$.

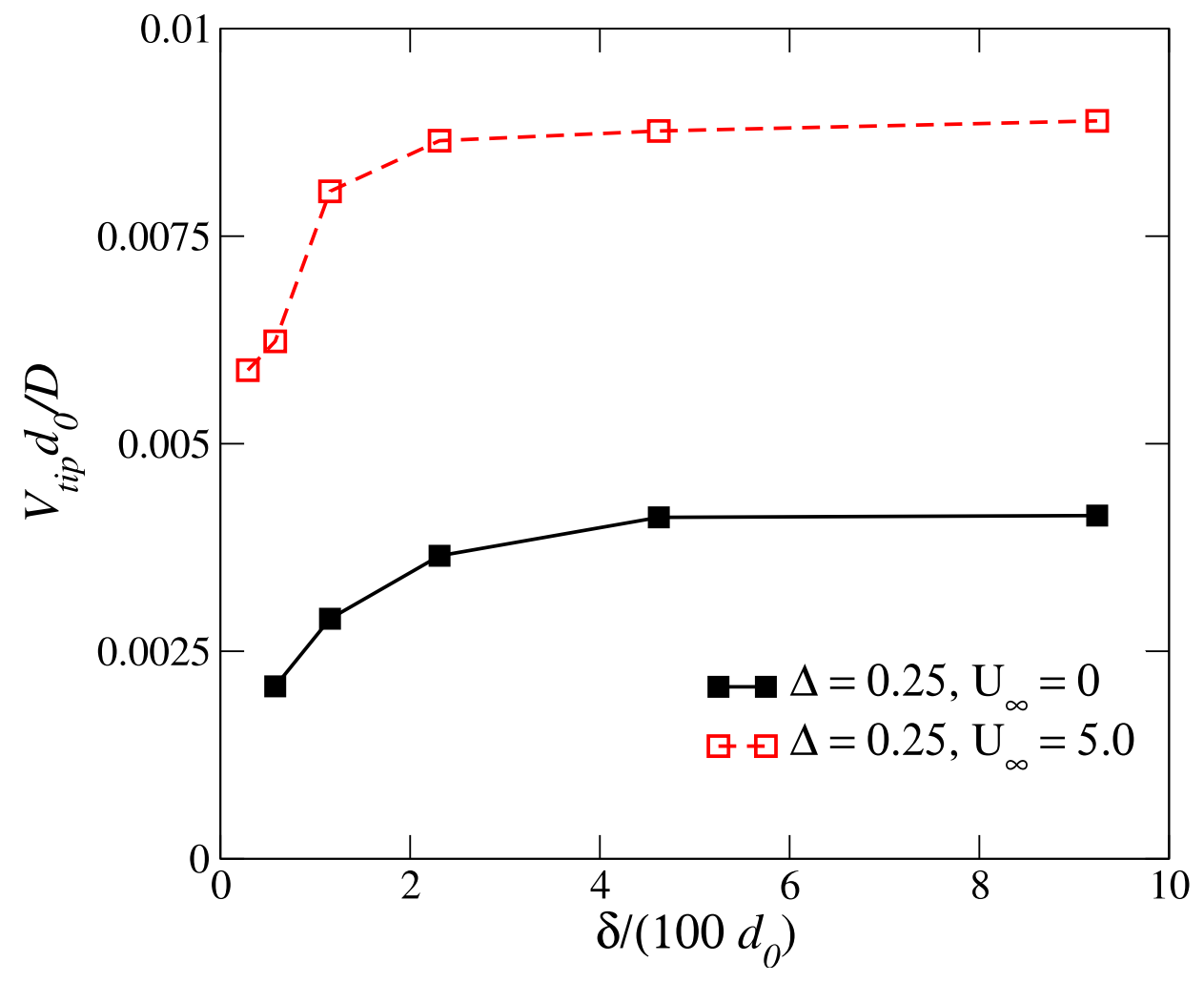

FIG. 2: Tip velocity vs. $\delta$, corresponding to cases 3 and 4 in Table 1.

In these plots, $\delta$ and $\rho_{t i p}$ were made dimensionless by scaling them with $d_{0}$, and $V_{t i p}$ is scaled by $D / d_{0}$. We compute the tip radii $\rho_{x z}$ and $\rho_{x y}$ along two principal planes using the method of Jeong et al. [18], and estimate the mean tip radius by the formula

$$
\rho_{t i p}=2\left(\frac{1}{\rho_{x y}}+\frac{1}{\rho_{x z}}\right)^{-1} .
$$

We can see from Fig. 2 that for large values of $\delta$, the tip velocity remains relatively unaffected by the box height. However, as we go to very small heights $V_{t i p}$ decreases quite dramatically. As $\delta$ is decreased, there is also a gradual decrease in the tip radius $\rho_{t i p}$. Clearly enough, box height has a pronounced effect on tip dynamics. Fluid flow induces a parallel shift in these curves. The dendrite tip velocity increases uniformly in the presence of flow [18]. On the other hand, the tip radius is lower than the case with pure diffusion.

The observed trends can be explained as follows. As long as $\delta$ is sufficiently large, the thermal field enveloping the dendrite will interact with the upper boundary at a distance that is relatively far behind the tip. In particular, the thickness of the thermal boundary layer near the tip remains unaffected by this interaction. However, as $\delta$ is decreased, this thickness can grow quite rapidly. We illustrate this effect by examining the temperature profile in the $x-z$ plane, as shown in Fig. 4 It is evident that the temperature contours are more spread out in Fig. 4(b) where $\delta=8$, compared to those in 4(a) where $\delta=64$. The increased boundary layer thickness, decreases the thermal gradient into the liquid at the liquid-solid interface, which in turn retards the growth rate as a direct consequence of the Stefan condition. Due to the zero flux boundary condition on the plane $z=\delta$, further reduction in $\delta$ makes heat transfer in the vertical direction almost completely ineffective. Tip curvature in the $x-z$ plane vanishes and the dendrite switches morphology from 3-D to 2-D. We note that once the dendrite goes 2-D, $\rho_{\text {tip }}=\rho_{x y}$.

An interesting result here is the 3-D to 2-D transition. We have performed tests with finer meshes (more elements in the vertical direction) to ensure that it is not simply an artifact of poor grid resolution. We believe this phenomenon 


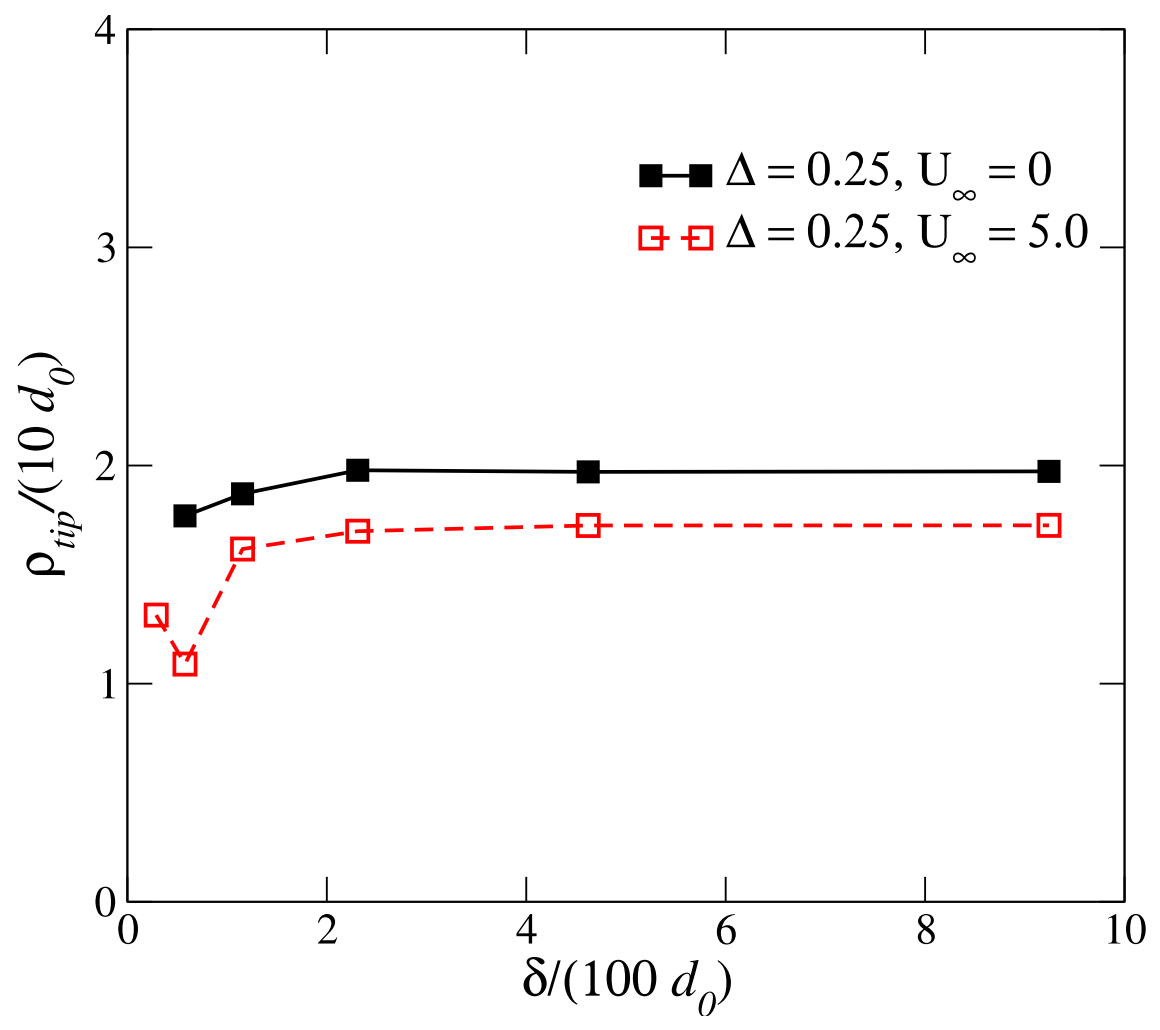

FIG. 3: Tip radius vs. $\delta$, corresponding to cases 3 and 4 in Table 1.

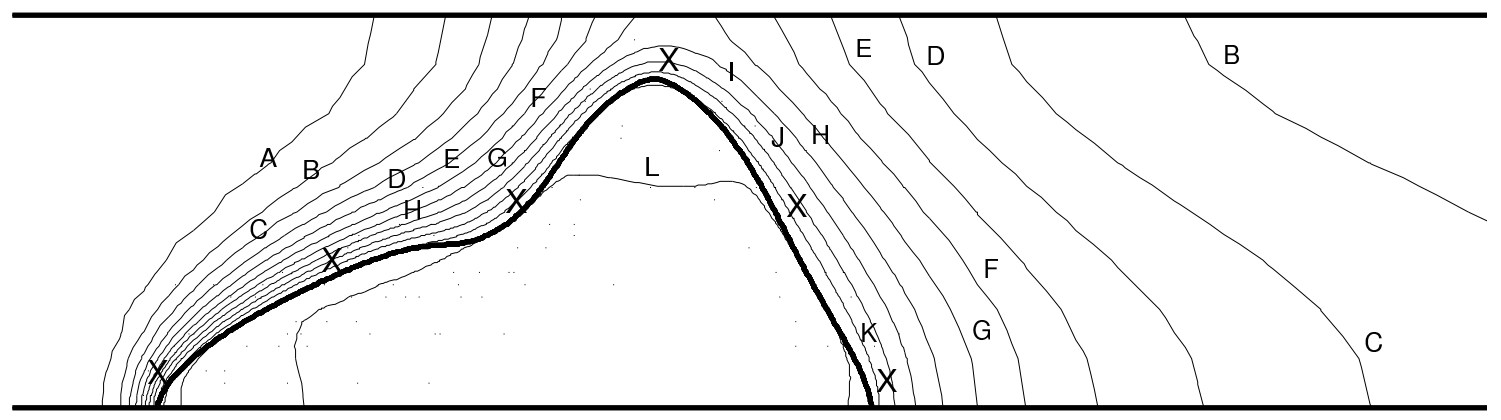

(a) $\delta=64$ corresponding to Case 4 (b). $u$ ranges from $\mathrm{A} \equiv-0.24$ to $\mathrm{L} \equiv 0.008$

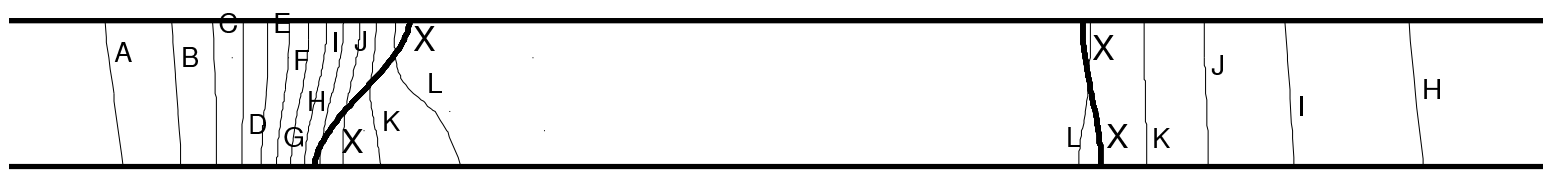

(b) $\delta=8$, corresponding to Case $4(\mathrm{e}) . u$ ranges from $\mathrm{A} \equiv-0.24$ to $\mathrm{L} \equiv 0.008$

FIG. 4: Temperature contours for two different box heights when $\Delta=0.25$ and $U_{\infty}=5.0$. In each case, the letter X symbolizes the dendrite outline (bold contour), under steady growth conditions. Contours are plotted in intervals of 0.025 . Notice that the contours near the dendrite tip are more spread out when $\delta$ is smaller. 


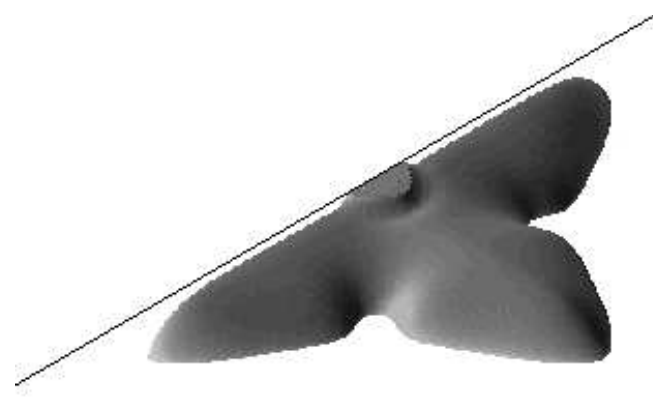

(a) $\delta=16,3-\mathrm{D}$ dendrite

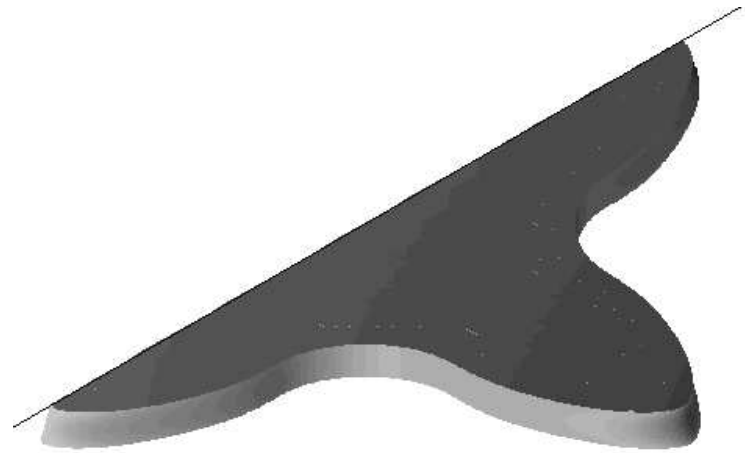

(b) $\delta=4,2-\mathrm{D}$ dendrite

FIG. 5: 3-D to 2-D dendritic transition at small $\delta$. The shaded surface is the dendrite $(\phi=0)$. For these runs $\Delta=0.55$ and $U_{\infty}=0$.

to be a consequence of the $\nabla \phi \cdot \mathbf{n}=0$ boundary condition on the upper boundary. The only way for the solid-liquid interface to match this boundary condition at small $\delta$ is for the curvature in the $x$ - $z$ plane to vanish. This is illustrated in Fig. [5]

It is also interesting to observe the effect that melt flow has on the interaction between the tip and the boundary. We find that melt convection reduces the value of $\delta$ where the tip velocity deviates from its nominal value. A straightforward explanation for this effect is that advection increases the rate of heat transport from the upstream dendrite arm. This increases the growth rate (hence $V_{t i p}$ ), while compressing the boundary layer, whose thickness scales as $D / V_{t i p}$. Thus the dendrite remains three dimensional for a smaller value of $\delta$ than was previously possible, with convection absent. A more negative value of the undercooling also has qualitatively the same effect on the strength of the tip-boundary interaction, since $V_{\text {tip }}$ again increases in this case. Fig. [6] summarizes these observations succinctly. Both melt convection and larger undercooling, cause points on respective curves whereupon interaction effects become important, to shift to the left.

Using a graph such as Fig. 6] one can derive a semi-quantitative estimate as to when the operating state becomes affected by the finite height of the container. If one assumes that the tip velocity at $\delta=128$ for each case is approximately the tip velocity of a dendrite growing under identical conditions in an infinite domain, it is possible to quantify the influence on the operating state in terms of a percentage deviation in the true tip velocity from this nominal value. If, for example, we consider deviations of the order of $3 \%$ to constitute a change in the operating state, a least squares fit to these cut-off points on the respective curves in Fig. [6 yields the criterion

$$
\delta \geq 0.7912\left(\frac{D}{V_{t i p}}\right)^{0.9242} .
$$

If this condition is not satisfied, then it is likely that the operating state is being influenced by the boundary. A simple condition such as the one in Eqn. (3) may be used as a rule of thumb in determining if experimental studies on free dendrite growth, in geometries similar to ours, are free from contamination. In fact, one may have intuitively guessed a condition of the type $\delta \geq \alpha\left(D / V_{t i p}\right)$ (where $\alpha$ is some constant) to apply based on physical arguments alone, and Eqn. (3) supports this conjecture.

\section{B. Non-wetting boundary conditions}

To underscore the importance of phase field boundary conditions in selecting a particular growth state, we present results from another simulation with $\Delta=0.55, U_{\infty}=0$ and $\delta=4$. This time we impose $\phi=-1$ on the upper boundary, which corresponds to a physical situation where the solidifying material is not allowed to wet the surface. A three dimensional surface plot of a steadily growing dendrite is shown for this case in Fig. 7

As expected, the dendrite does not adhere to the top surface at all. We conclude that this will be the case for any $\delta$ if Dirichlet conditions of this nature are imposed. It is evident then, that the 3D-2D transition that we saw 


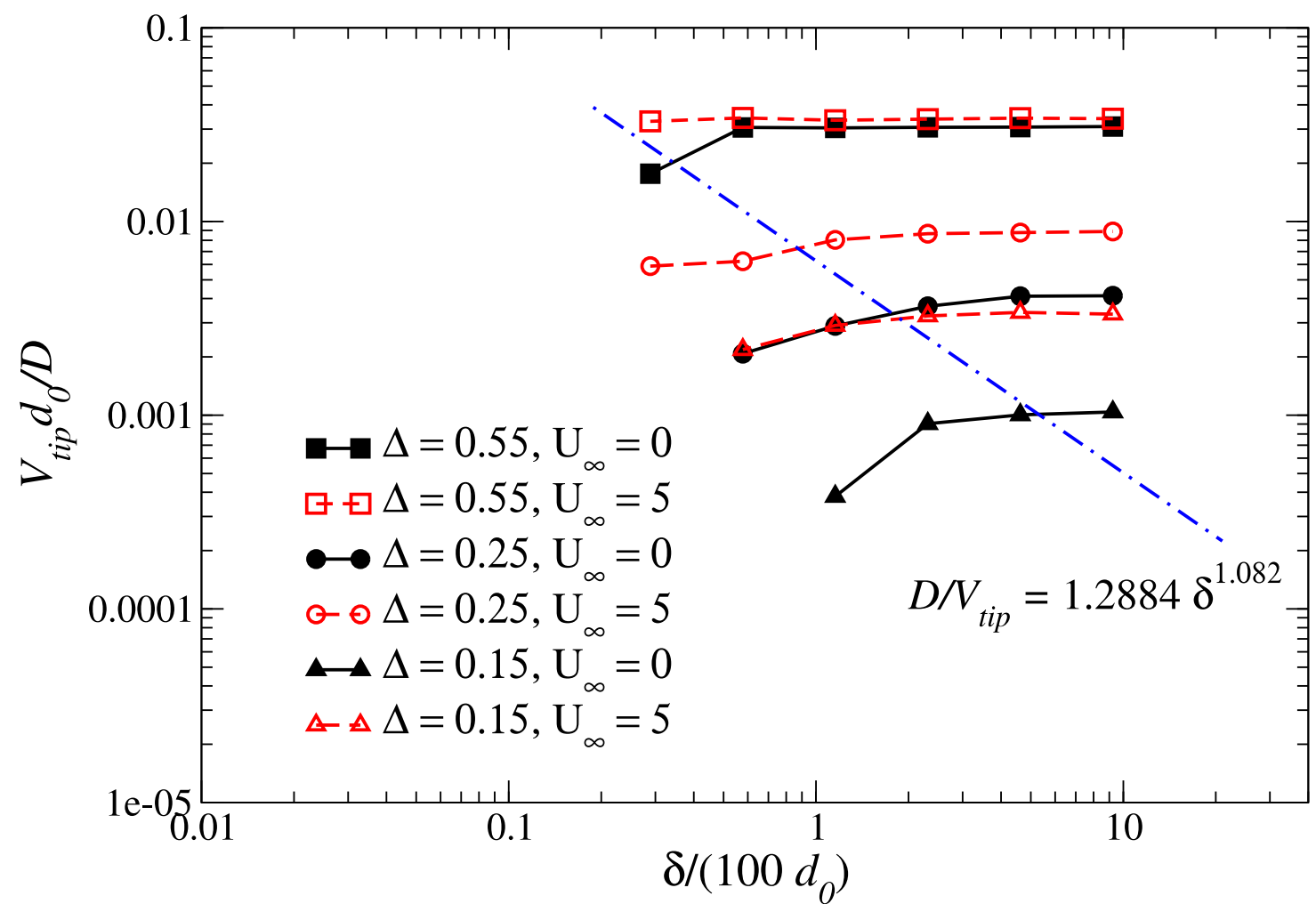

FIG. 6: Tip velocity as a function of $\delta$ for different undercooling and flow conditions. A weak power law relationship emerges between the $\delta$ below which interaction effects are strong, and diffusion length $D / V_{\text {tip }}$.

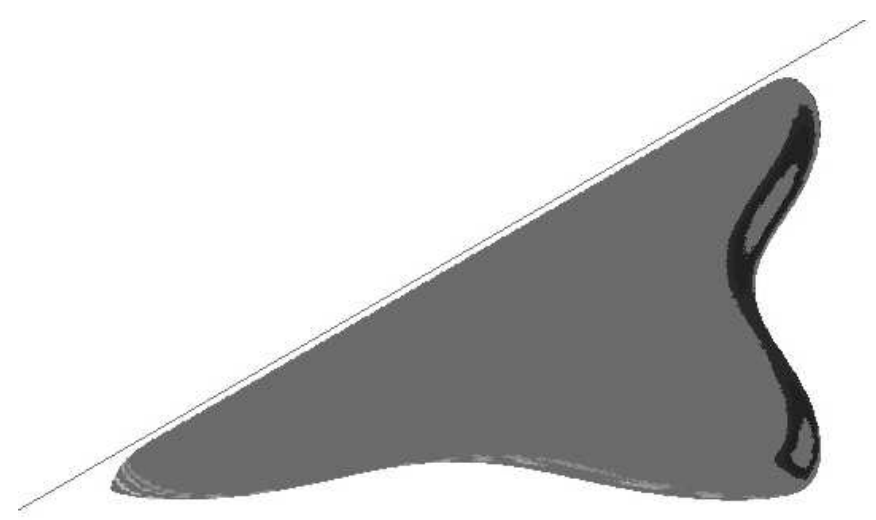

FIG. 7: Three dimensional dendritic growth for $\Delta=0.55, U_{\infty}=0$ and $\delta=4$, with $\phi=-1$ on the upper boundary. Note that the dendrite does not wet this surface.

previously is a strong function of the boundary conditions imposed on the phase field. It would be interesting to conduct a detailed investigation of the effect of different boundary conditions on the tip in 3-D. The interested reader is referred to the article by Sémoroz et al. [30], which discusses the influence of different contact angles on a dendrite's tip velocity, in two-dimensional thin film solidification.

\section{EFFECT OF SMALL $\delta$ IN A DIRECTIONALLY SOLIDIFIED ALLOY}

In this section, we describe results from our simulations on a directionally solidified alloy, and make comparisons with the experimental data of Liu et al. 22]. These simulations were conducted for the range of pulling speeds and 
Table 2. Alloy simulations

\begin{tabular}{lll}
\hline \hline Case & $V_{p}$ & $\delta$ \\
\hline 1 & 0.8 & $64,32,16,8,4$ \\
2 & 1.0 & $64,32,16,8,4$ \\
3 & 1.5 & $64,32,16,8,4$ \\
4 & 2.0 & $64,32,16,8,4$ \\
\hline
\end{tabular}

Table 3. Physical properties of a SCN-Salol alloy system

\begin{tabular}{ll}
\hline$|m|$ (Liquidus slope) & $0.7 \mathrm{~K} / \mathrm{wt} . \%$ \\
$D$ (Diffusion coefficient) & $8 \times 10^{-10} \mathrm{~m}^{2} / \mathrm{s}$ \\
$\Gamma$ (Gibbs-Thomson coefficient) & $0.64 \times 10^{-7} \mathrm{~K} \mathrm{~m}$ \\
$k$ (Partition coefficient) & 0.2 \\
$G$ (Thermal gradient) & $4 \mathrm{~K} / \mathrm{mm}$ \\
$d_{0}$ (Capillary length) & $3.265 \times 10^{-8} \mathrm{~m}$ \\
$l_{T}$ (Thermal length) & $4.9 \times 10^{-4} \mathrm{~m}$ \\
\hline
\end{tabular}

channel depths shown in Table 2. The depth $\delta$ was varied from 64 to 4, and in each case, the simulation was continued until the interface became stationary in the moving frame. From the stable array of cells formed, a few were chosen as representative of the array, their tip radii were extracted in the two principal planes using a least squares quadratic polynomial fit, and the mean tip radius of each cell was computed using Eqn. (2). An average over these values was taken to be the mean tip radius of the interface. We note that this process required some approximation, especially in cases where we found steadily growing cells of disparate sizes that would have made such averaging inappropriate. In such cases, our usual approach was to choose cells farthest from the boundaries, and where even this failed to provide clear-cut choices, smaller cells were chosen because of their lesser likelihood to split. In a majority of our runs however, the choices were unambiguous. For certain values of $\delta$ we found interface evolution to occur in a way that cells would creep on either the top or bottom surfaces. In those cases, the phase-field boundary conditions on the respective surfaces allowed us to treat them as symmetry planes for the purpose of calculating tip curvature.

\section{A. Selection of simulation parameters}

The following values for the lateral dimensions were seen to yield satisfactory results. $L_{x}=L_{y}=256$ when $\delta \leq 16$, and $L_{x}=256, L_{y}=128$, otherwise. We chose our simulation parameters to keep computations tractable. It took about 90 hours of CPU time on a $3.1 \mathrm{GHz}$ processor to simulate a typical directional solidification experiment for a chosen set of phase-field parameters on a mesh with about 170000 elements $(\delta=64)$. The interface required about 250 dimensionless time units to reach steady state in this case. As noted earlier, the use of a moving reference frame allowed us to cut substantial costs associated with the need for larger domains to prevent the diffusion field from running out of the domain.

We did not attempt to model a real material in this study as this caused our simulations to become considerably more expensive. To illustrate this, consider a SCN-Salol system having the properties listed in Table 3. The conditions in a directional solidification experiment are completely described by the following two dimensionless control parameters, $M=d_{0} / l_{T}=6.66 \times 10^{-5}$ and $S=V_{p} d_{0} / D=2.04 \times 10^{-4}$, where $d_{0}$ is the capillary length, $l_{T}$ is the thermal length and $D$ is the solute diffusivity in the liquid phase; for a pulling velocity of $V_{p}=5 \mu \mathrm{m} / \mathrm{s}$. To get converged results with the phase-field model we require that the solution become independent of the parameter $\epsilon=W_{0} / d_{0}$. After ensuring vanishing interface kinetics, the following relationships involving the phase-field parameters are realized: $D \tau_{0} / W_{0}^{2}=a_{1} a_{2} \epsilon, V_{p} \tau_{0} / W_{0}=S a_{1} a_{2} \epsilon^{2}$, and $l_{T} / W_{0}=1 /(\epsilon M)$. Here, $a_{1}=0.8839$ and $a_{2}=0.6267$, are constants that arise in the phase-field formulation. [28]

Echebarria et al. [28] have shown that mesh converged results can be obtained with $\epsilon$ as large as 50. Setting $\epsilon=50$, gives us an under-determined system of three equations with the five unknowns $D, V_{p}, l_{T}, \tau_{0}$ and $W_{0}$. Making arbitrary choices for two of these parameters by setting $W_{0}=\tau_{0}=1$, we obtain $D=27.7, V_{p}=0.2825$, and $l_{T}=300$. A large value of $l_{T}$ implies $L_{x}$ needs to be very large at steady state, even in a moving reference frame, to contain the diffusion field. To avoid this, if we choose a more tractable value for $l_{T}$ (say 100), and fix $\tau_{0}=1$, we now get 
$W_{0}=0.3333, D=3.077$ and $V_{p}=0.094$. Thus, the smallest element in our mesh needs to be at the very least $\Delta x=0.3333$. Stability considerations now place a severe restriction on the size of the time step $(\Delta t)$ needed for solving the phase-field equations by the Forward-Euler method, as $\Delta t \sim \Delta x^{2}$. Since it is clearly impossible to choose both $l_{T}$ and $W_{0}$ independently, calculations involving real materials are typically more expensive.

We choose instead a more computationally favorable set of dimensionless parameters $M$ and $S$ for our study. To achieve our primary objective, which is to study the effect of $\delta$ on interface morphology, we anticipate, and in the following paragraphs demonstrate, that this hypothetical treatment will not obscure any physics. The parameters used in our study are: $\tau_{0}=W_{0}=1, D=20, k=0.8, \epsilon_{4}=0.05$, and $l_{T}=|m|(1-k) C_{\infty} / k G=50$; where $m$ is the liquidus slope, $G$ is the imposed thermal gradient, and $C_{\infty}$ is the far field solute concentration. The condition for negligible interface kinetics gives $d_{0}=0.0277$ and therefore $\epsilon=36.1$, which is sufficiently small to ensure convergence. The size of the smallest element in our adaptive mesh is $\Delta x=1$, when $\delta \geq 8$, and $\Delta x=0.5$, when $\delta=4$, while $\Delta t=0.005$ is the size of the time-step. For these parameter choices, the dimensionless control parameters work out to be $M=5.54 \times 10^{-4}$ and $S \sim 1.385 \times 10^{-3}$.

\section{B. Interface morphology and comparison with experiments}

In order to test the model, we initially performed a set of runs to verify the Mullins and Sekerka stability limit of a planar interface [31], perturbed by small sinusoidal perturbations. We found that the model captures the stability spectrum correctly. Having convinced ourselves that this fundamental requirement was met, we proceeded with our study. Comparisons with the experimental data in this section offer a better validation of the model.

Fig. [8] shows the computed interface morphology at different values of $\delta$. For small values of pulling speed $\left(V_{p} \leq 2\right)$, the steady state consists of a stationary array of cells as in Fig. 8 However, unlike the cells observed in experiments, that are usually characterized by blunt tips, these appear to have sharper and better defined tips, giving the impression of dendrites. It is conceivable that ignoring thermal noise in our calculations is responsible for the absence of sidebranches on these structures, that are typical of dendrites. At large values of $\delta$, we find that the tip radii of these cells, measured on the two principal planes, are almost identical. However, as $\delta$ decreases, the in plane radii diverge from one another. In particular, the radius in the $x-z$ plane becomes significantly smaller, and cross sections of the cells look elliptic. At $\delta=4$, for small pulling speeds $\left(V_{p} \leq 1\right)$, we get a two-dimensional interface (Fig. 8(c) . The inter-cellular spacing also increases as $\delta$ is decreased.

As pulling velocity is increased, the morphology becomes finer, with sharper and more tightly packed cells. This behavior is consistent with that seen of both cellular and dendritic arrays in directional solidification experiments [21, 22, 32], where the primary spacing decreases with $V_{p}$.

For the set of phase-field parameters we have chosen, if $V_{p} \geq 2.5$, the interface does not reach steady state in a reasonable amount of CPU time, due to repeated tip-splitting of the cells. Splitting is initiated by oscillations that appear at the tip and propagate downward along the trunk of the cell. Cell spacing and shapes change very rapidly in this regime. We did not continue these runs any further to check if steady state is reached eventually. Instead, we set $V_{p}=2.0$ as an upper bound on the pulling velocity, below which a stationary state was always the outcome. Fortunately, this still left us with sufficient sample space to conduct our study and make effective comparisons.

To enable plotting of our results on the same graph with the experimental data of Liu et al., which corresponds to a SCN - $0.7 \%$ wt. Salol system (properties in Table 3), we non-dimensionalized the axes as follows. The abscissa is the pulling speed (i.e. the tip velocity at steady state) $V_{t i p}$, scaled by a characteristic velocity $D / d_{0} k$, while the ordinate is the tip radius $\rho_{t i p}$, scaled by the diffusion length $D / V_{t i p}$. One may appreciate the fact that the abscissa is in fact the dimensionless parameter that we had earlier denoted by $S$, multiplied by $k$. Fig. 9 shows a comparison of the data. The open symbols correspond to the experimental data of Liu et al., while the solid symbols correspond to our calculations. A comparison between our data and theirs holds up surprisingly well. Of special significance are the following two observations: 1) Although we conducted our simulations at values of $S$ and $M$ that were each about an order of magnitude off theirs, the two sets of data correlate very well, i.e. appear to collapse on parallel curves that are not significantly different by way of intercept. This tells us that our choices of parameters for scaling the axes are appropriate. 2) Since their experimental data correspond to dendritic arrays, the cell-like structures we have computed are likely branchless dendrites.

In their experiments, Liu et al. note that tip radius data for dendritic arrays agree quite nicely with a relationship of the form $\rho_{\text {tip }}^{2} V_{t i p}=C$, where $C$ is a constant dependent on $d_{0}, k$ and $D$, as postulated by theoretical models of constrained growth [19, 20]. However, when $\delta$ is of the order of inter-dendritic spacing $\lambda_{1}$, this agreement deteriorates. This is evident in Fig. 9] where the $\rho_{\text {tip }}^{2} V_{\text {tip }}=C$ line is shown as a guide to the eye. The open circles, which are data for $\delta=12.5 \mu \mathrm{m}$ deviate in both slope and intercept from this line, which passes through the rest of their data, indicating a breakdown in the relationship. We observe similar trends in our data, viz., the line $\rho_{\text {tip }}^{2} V_{t i p}=C$ fits our data at $\delta=32$ and 64 reasonably well, but as $\delta$ decreases from 16 to 4 , this agreement deteriorates. 


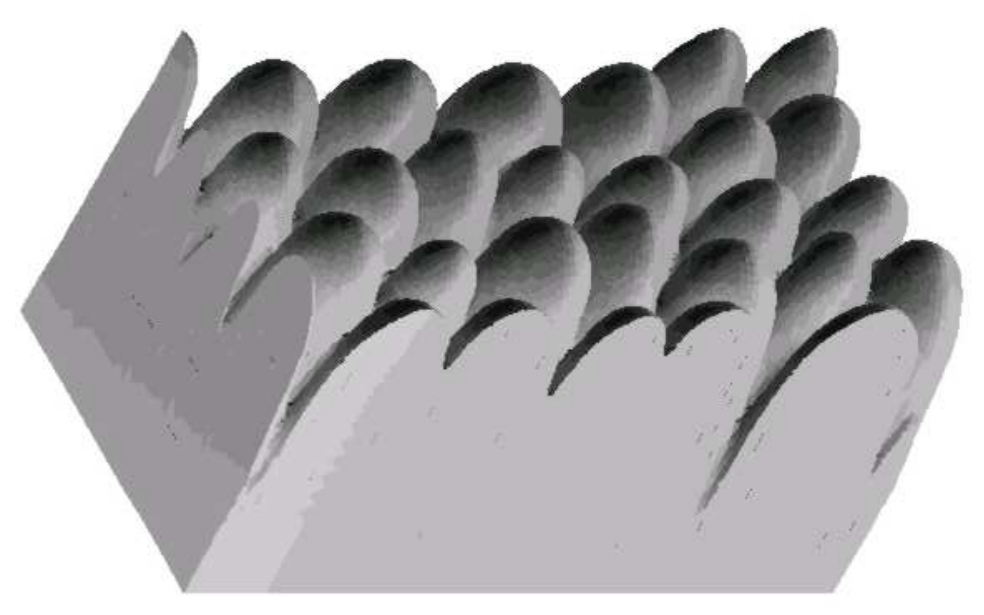

(a) $\delta=64, V_{p}=1.5$, three-dimensional cells

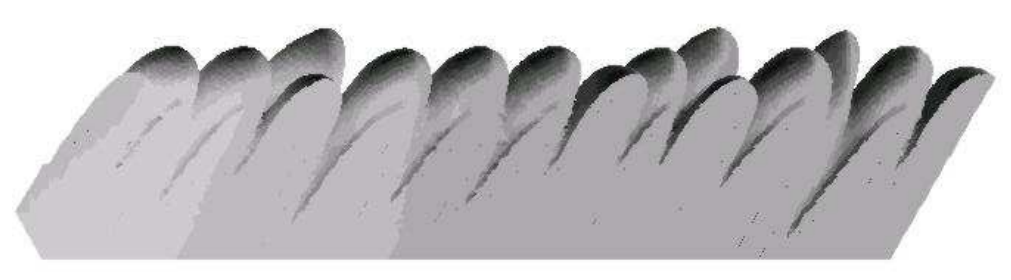

(b) $\delta=16, V_{p}=1.5$, three-dimensional cells

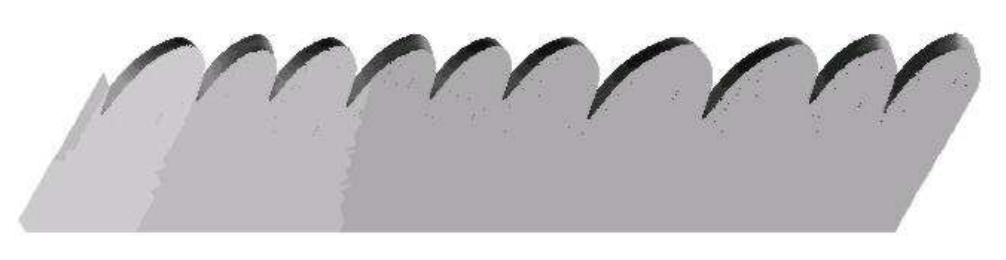

(c) $\delta=4, V_{p}=0.8$, two-dimensional cells

FIG. 8: Interface morphology in a directionally solidified alloy. At steady state, stable arrays of three-dimensional cells appear. Note that at $\delta=4$, the array comprises of two-dimensional cells.

As in the numerical experiments with the pure material, we find that decreasing $\delta$ has a pronounced effect on interface morphology. Dendritic arrays seen in experiments have a certain structure/periodicity to them, that arises from underlying crystalline symmetries. For example, in our simulations we observe that the cells constitute a hexagonal array. When $\delta$ is large, away from the boundaries the diffusion field surrounding each cell tip obeys this symmetry, and the optimal $\lambda_{1}$ is selected. As $\delta$ decreases however, the diffusion field becomes increasingly asymmetric due to interaction with the boundaries at $x=0$ and $x=\delta$. In particular, solute rejection decreases in the vertical 


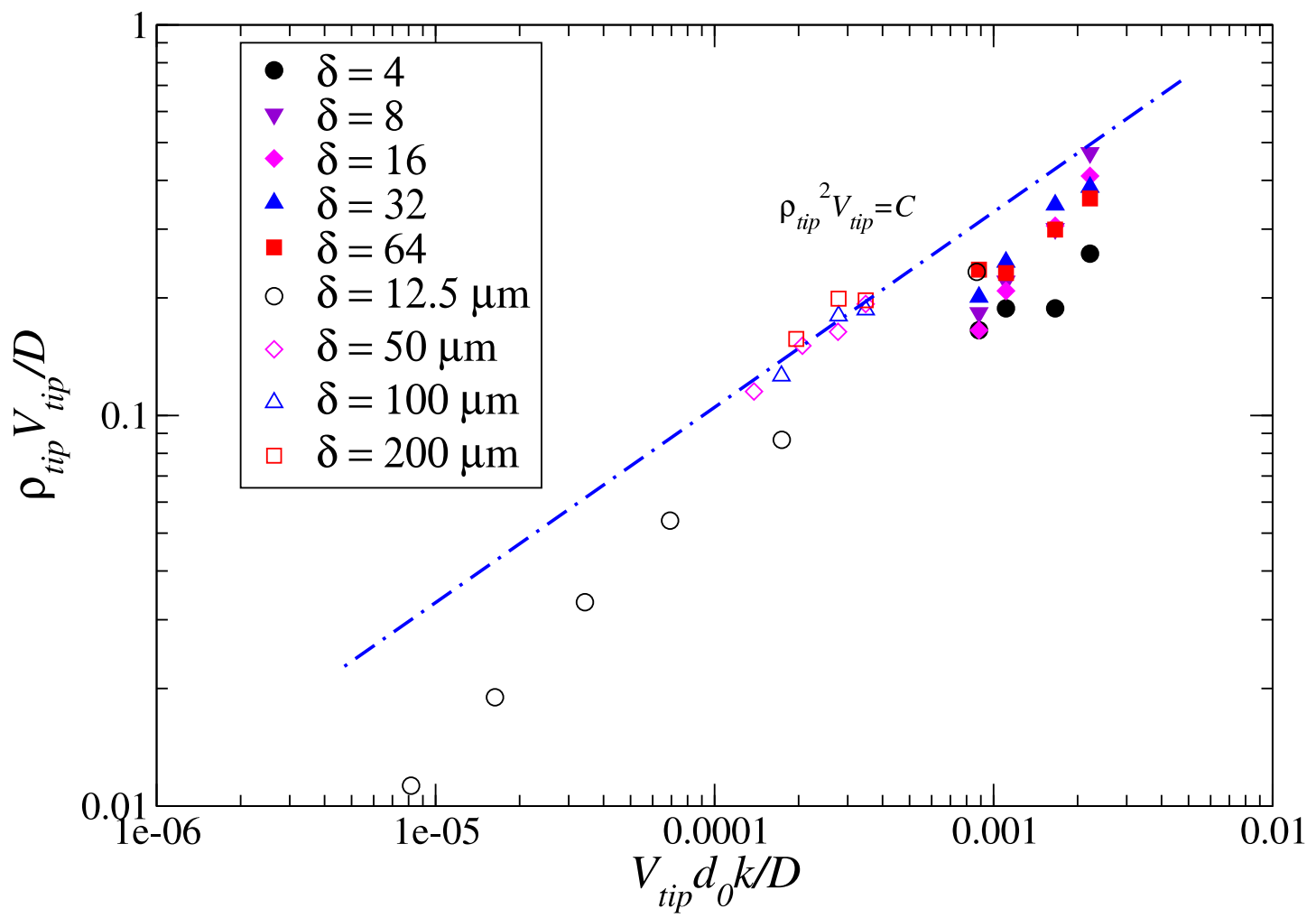

FIG. 9: Comparison of binary alloy simulations with the phase-field model, and experimental data of Liu et al. 22]. Solid symbols correspond to the phase-field model and the open symbols are experimental data. The line denotes a relationship of the form $\rho_{t i p}^{2} V_{t i p}=C$ between tip radius and velocity, where $C$ can be expressed in terms of process parameters.

plane $x$ - $z$, while increasing in the horizontal plane $x-y$. Increased solute accumulation between cells in $x-y$, contributes to an increase in $\lambda_{1}$. However, since $V_{t i p}$ is fixed by the pulling speed, and a certain rate of solute rejection needs to be maintained, the tips tend to grow sharper as $\lambda_{1}$ increases. It is precisely this effect that causes the operating state to deviate from theoretical predictions.

To check for what value of $\delta$ our results deviate from theory, let us assume the steadily growing array at $\delta=64$, $V_{p}=1.5$ (Fig. 8(a) to be one in which cells close to the plane $z=32$ are "free" from boundary effects. We estimate the cell spacing in that plane to be $\lambda_{1}^{f} \approx 18$, where the superscript 'f' denotes "free". When $\delta=16$ and $V_{p}=1.5$ (Fig. 8(b)], we notice that our data points start departing from the theoretical prediction, viz. we see a relationship of the type $\rho_{t i p}^{a} V_{t i p}=C$, where $a>2$. This suggests that agreement with theory deteriorates as $\delta \sim \lambda_{1}^{f}$, which is what Liu et al. concluded from their experiments. A more precise form of the above criterion can be obtained my making a careful study of $\lambda_{1}$ as a function of $\delta$, for different $V_{p}$, and obtaining a criterion based on a least squares fit to the deviation points (as we did with the pure material). Increasing pulling velocity suppresses tip-boundary interaction by reducing the thickness of the diffusion boundary layer $D / V_{t i p}$, similar to the effect of melt convection in pure materials, and should induce a leftward shift in the curves.

\section{CONCLUDING REMARKS}

We have investigated the role of confinement on solidification in both pure materials and binary alloys. Our simulations show that, for equi-axed growth in a pure material, the dendrite's operating state is affected when the container dimension $\delta$, approaches the scale of the diffusion field $D / V_{\text {tip }}$ near the tip. For directionally solidified binary alloys, confinement effects become important when $\delta$ is of the order of the primary dendrite spacing $\lambda_{1}$. Where applicable, one needs to consider the influence of these interactions when comparing experimental data with theoretical models that do not account for confinement effects.

It is notable that we were able to make meaningful comparisons with real experimental results using the phase-field model for the alloy. In particular, the agreement obtained in the trends shown by the dendrite tips for different $\delta$ and 
$V_{p}$ is very encouraging, and is a testament to the power of phase-field modeling. Some ambiguity remains in classifying the computed microstructure as cells or dendrites. Since our results correlated well with dendrite data, we would like to think of them as dendrites. Perhaps, incorporating random fluctuations in the phase-field model will resolve this issue. We also found tip-splitting inducing oscillations above certain values of the pulling speed. We are unsure as to whether this instability has a physical meaning. In experiments, it is seen that increasing $V_{p}$ causes a decrease in $\lambda_{1}$ and $\rho_{\text {tip }}$ for a steadily growing interface, and our simulations capture this effect, viz. as $V_{p}$ increases the cells split in a manner that produces a more stable configuration with a smaller $\lambda_{1}$ and $\rho_{\text {tip }}$. However, when $V_{p} \geq 2.5$, it appears that an optimal configuration is not possible in our system, given the constraints on its size and boundary conditions. We speculate that larger domains should allow for more stable cell configurations at higher pulling speeds. This issue too needs further investigation.

We observed a change in dimensionality of the liquid-solid interface for certain values of $\delta$ and $V_{\text {tip }}$, when zero flux boundary conditions were imposed on the phase-field variable. There is some experimental evidence of this phenomenon in the literature. Liu and Kirkaldy 32] reported a 2-D to 3-D transition in their experiments on a SCN-Salol mixture. In their directional solidification experiments in a cell of fixed height $(\delta=28 \mu \mathrm{m})$, they found this transition to occur at a driving velocity of $10.8 \mu \mathrm{m} / \mathrm{sec}$. At a lower driving velocity of $7.6 \mu \mathrm{m} / \mathrm{sec}$, the dendrites looked two dimensional. In our analysis of directional solidification, we found at $\delta=4$, the cells underwent a 2-D to $3-\mathrm{D}$ transition as the pulling velocity was changed from 1 to 1.5. The significance of this result is that through an appropriate selection of $\delta$ and $V_{p}$ in experiments, it should be possible to obtain almost two dimensional dendritic arrays in materials that favor wetting. Such experiments will permit more favorable comparisons with 2-D dendrite growth theories, since finite boundary effects along the $z$ axis cease to impact the growth.

\section{Acknowledgments}

The authors gratefully acknowledge support for this work from NASA under Grant NAG 8-1657, and from the National Science Foundation under Grant DMR 01-21695. We thank Jun-Ho Jeong and Navot Israeli for their significant contributions in the development and implementation of the adaptive grid algorithm which made this study possible, and the Computational Science and Engineering program at UIUC for availing us their computing facilities.

[1] G. P. Ivantsov, Dokl. Akad. Nauk USSR 58, 1113 (1947).

[2] D. E. Temkin, Dokl. Akad. Nauk SSSR 132, 1307 (1960).

[3] J. S. Langer and H. Müller-Krumbhaar, J. Cryst. Growth 42, 11 (1977).

[4] J. S. Langer and H. Müller-Krumbhaar, Acta Metall. 26, 1681 (1978).

[5] R. Brower, D. Kessler, J. Koplik, and H. Levine, Phys. Rev. Lett. 51, 1111 (1983).

[6] E. Ben-Jacob, N. Goldenfeld, J. Langer, and G. Schön, Phys. Rev. Lett. 51, 1930 (1983).

[7] E. Ben-Jacob, N. Goldenfeld, B. Kotliar, and J. Langer, Phys. Rev. Lett. 53, 2110 (1984).

[8] A. Karma and W.-J. Rappel, Phys. Rev. E. 53, 3017 (1995).

[9] M. E. Glicksman and R. J. Schaefer, J. Cryst. Growth 1, 297 (1967).

[10] S.-C. Huang and M. Glicksman, Acta Metall. 29, 1697 (1981).

[11] M. B. Koss, M. E. Glicksman, A. O. Lupulescu, L. A. Tennenhouse, J. C. LaCombe, D. C. Corrigan, J. E. Frei and D. C. Malarik, 36th Aerospace Sciences Meeting, January 12-15, 1998, Reno, NV, AIAA-98-0809.

[12] N. Provatas, J. Dantzig, and N. Goldenfeld, Phys. Rev. Lett. 80, 3308 (1998).

[13] N. Provatas, J. Dantzig, and N. Goldenfeld, J. Comp. Phys. 148, 1 (1999).

[14] W. N. Gill, Y. W. Lee, K. K. Koo, and R. Ananth, in Interactive Dynamics of Convection and Solidification, edited by S. H. Davis, H. E. Huppert, U. Müller, and M. G. Worster (Kluwer Academic Publishers, 1992).

[15] Y.-W. Lee, R. Ananth, and W. N. Gill, J. Cryst. Growth 132, 226 (1993).

[16] Ph. Bouissou and B. Perrin and P. Tabeling, Phys. Rev. A 40, 509 (1989).

[17] D. A. Saville and P. J. Beaghton, Phys. Rev. A 37, 3423 (1988).

[18] J.-H. Jeong, N. Goldenfeld, and J. A. Dantzig, Phys. Rev. E 64, 041602 (2001).

[19] R. Trivedi, J. Cryst. Growth 49, 219 (1980).

[20] W. Kurz and D. J. Fisher, Fundamentals of Solidification (Trans Tech Publications, 1989), $3^{\text {rd }}$ ed.

[21] K. Somboonsuk, J. T. Mason, and R. Trivedi, Met. Trans. A 15A, 967 (1984).

[22] S. Liu, R. Trivedi, B. P. Athreya, and J. A. Dantzig, unpublished work.

[23] B. P. Athreya and J. A. Dantzig, in Solidification Processes and Microstructures: A symposium in honor of Wilfried Kurz, edited by M. Rappaz, C. Beckermann, and R. Trivedi (TMS-AIME, 2004), pp. 357-368.

[24] J. S. Langer, Directions in Condensed Matter Physics (World Scientific, Singapore, 1986), vol. 1, p. 165.

[25] C. Beckermann, H.-J.Diepers, I. Steinbach, A. Karma, and X. Tong, J. Comp. Phys. 154, 468 (1999). 
[26] A. Karma, Phys. Rev. Lett. 87, 115701 (2001).

[27] J. C. Ramirez, C. Beckermann, A. Karma, and H. J. Diepers, Phys. Rev. E 69, 051607 (2004).

[28] B. Echebarria, R. Folch, A. Karma, and M. Plapp, Phys. Rev. E 70, 061604 (2004).

[29] P. M. Gresho, S. T. Chan, M. A. Christon, and A. C. Hindmarsh, Int. J. Numer. methods fluids 21,837 (1995).

[30] A. Sémoroz, S. Henry, and M. Rappaz, Met. Trans. A 31A, 487 (2000).

[31] W. W. Mullins and R. F. Sekerka, J. Appl. Physics 35, 444 (1964).

[32] L. X. Liu and J. S. Kirkaldy, J. Cryst. Growth 140, 115 (1994). 